\title{
CYANIDES AND ISOCYANIDES OF FIRST-ROW TRANSITION METALS: MOLECULAR STRUCTURE, BONDING, AND ISOMERIZATION BARRIERS
}

\section{Supporting Information}

Víctor M. Rayón ${ }^{1}$, Pilar Redondo ${ }^{1}$, Haydee Valdés ${ }^{2}$, Carmen Barrientos ${ }^{1}$, and Antonio Largo ${ }^{1 *}$

${ }^{1}$ Departamento de Química Física y Química Inorgánica Facultad de Ciencias Universidad de Valladolid 47005 Valladolid Spain

${ }^{2}$ Center for Biomolecules and Complex Systems Institute of Organic Chemistry and Biochemistry Academy of Sciences of Czech Republic 16610 Prague 6

Czech Republic

* Author to whom correspondence should be addressed. e-mail: alargo@qf.uva.es

Fax: 34-983-423013 
Table S1. Harmonic vibrational frequencies, IR intensities, and dipole moments for MCN species obtained with the B3LYP/6-311+G(3df) and QCISD/6-311+G(d) (second line) methods.

\begin{tabular}{|c|c|c|c|c|c|c|c|}
\hline & \multicolumn{6}{|c|}{ IR frequencies $\left(\mathrm{cm}^{-1}\right)$ and intensities $\left(\mathrm{km} \mathrm{mol}^{-1}\right)$} & \multirow{2}{*}{$\begin{array}{c}\text { Dipole moment } \\
\mu(\mathrm{D})\end{array}$} \\
\hline & $\begin{array}{c}v_{1} \\
(\mathrm{C}-\mathrm{N} \text { stretch })\end{array}$ & $\mathrm{I}_{1}$ & $\begin{array}{c}v_{2} \\
\text { (M-C stretch) }\end{array}$ & $\mathrm{I}_{2}$ & $\begin{array}{c}v_{3} \\
\text { (bend) }\end{array}$ & $\mathrm{I}_{3}$ & \\
\hline $\operatorname{ScCN}\left({ }^{3} \Delta\right)$ & $\begin{array}{l}2233 \\
2165\end{array}$ & $\begin{array}{l}34.96 \\
22.65\end{array}$ & $\begin{array}{l}398 \\
379\end{array}$ & $\begin{array}{l}69.45 \\
60.85\end{array}$ & $\begin{array}{l}169 \\
138\end{array}$ & $\begin{array}{l}0.15 \\
0.01\end{array}$ & $\begin{array}{c}6.73 \\
6.17\end{array}$ \\
\hline $\operatorname{TiCN}\left({ }^{4} \Phi\right)^{\mathrm{a}}$ & $\begin{array}{l}2220 \\
2132\end{array}$ & $\begin{array}{l}10.34 \\
16.04\end{array}$ & $\begin{array}{l}410 \\
395\end{array}$ & $\begin{array}{l}51.84 \\
41.50\end{array}$ & $\begin{array}{c}187,198 \\
163\end{array}$ & $\begin{array}{c}0.72,1.88 \\
0.20\end{array}$ & $\begin{array}{l}6.87 \\
6.26\end{array}$ \\
\hline $\operatorname{VCN}\left({ }^{5} \Delta\right)$ & $\begin{array}{l}2212 \\
2118\end{array}$ & $\begin{array}{l}0.41 \\
4.11\end{array}$ & $\begin{array}{l}418 \\
413\end{array}$ & $\begin{array}{l}34.66 \\
28.68\end{array}$ & $\begin{array}{l}219 \\
186\end{array}$ & $\begin{array}{l}1.56 \\
1.58\end{array}$ & $\begin{array}{l}7.11 \\
6.77\end{array}$ \\
\hline $\mathrm{CrCN}\left({ }^{6} \Sigma^{+}\right)$ & $\begin{array}{l}2224 \\
2141\end{array}$ & $\begin{array}{c}2.18 \\
10.83\end{array}$ & $\begin{array}{l}418 \\
401\end{array}$ & $\begin{array}{l}40.83 \\
46.06\end{array}$ & $\begin{array}{l}223 \\
189\end{array}$ & $\begin{array}{l}3.55 \\
8.76\end{array}$ & $\begin{array}{l}7.69 \\
8.62\end{array}$ \\
\hline $\operatorname{MnCN}\left({ }^{7} \Sigma^{+}\right)$ & $\begin{array}{l}2232 \\
2177\end{array}$ & $\begin{array}{l}52.50 \\
43.30\end{array}$ & $\begin{array}{l}388 \\
387\end{array}$ & $\begin{array}{l}92.91 \\
88.20\end{array}$ & $\begin{array}{l}157 \\
153\end{array}$ & $\begin{array}{l}3.71 \\
1.46\end{array}$ & $\begin{array}{l}4.86 \\
5.07\end{array}$ \\
\hline $\mathrm{FeCN}\left({ }^{6} \Delta\right)$ & $\begin{array}{l}2233 \\
2181\end{array}$ & $\begin{array}{l}76.85 \\
56.33\end{array}$ & $\begin{array}{l}408 \\
410\end{array}$ & $\begin{array}{l}90.30 \\
86.29\end{array}$ & $\begin{array}{l}169 \\
169\end{array}$ & $\begin{array}{l}4.87 \\
2.15\end{array}$ & $\begin{array}{l}4.27 \\
4.59\end{array}$ \\
\hline $\operatorname{CoCN}\left({ }^{3} \Phi\right)^{\mathrm{a}}$ & $\begin{array}{l}2234 \\
2120\end{array}$ & $\begin{array}{c}2.30 \\
16.17\end{array}$ & $\begin{array}{l}446 \\
443\end{array}$ & $\begin{array}{c}23.15 \\
7.81\end{array}$ & $\begin{array}{l}252,265 \\
142,261\end{array}$ & $\begin{array}{l}7.69,9.21 \\
0.73,8.01\end{array}$ & $\begin{array}{l}7.22 \\
7.27\end{array}$ \\
\hline $\operatorname{NiCN}\left({ }^{2} \Delta\right)$ & $\begin{array}{l}2249 \\
2215\end{array}$ & $\begin{array}{l}0.00 \\
8.80\end{array}$ & $\begin{array}{l}476 \\
450\end{array}$ & $\begin{array}{c}13.11 \\
1.21\end{array}$ & $\begin{array}{l}255 \\
224\end{array}$ & $\begin{array}{l}3.23 \\
4.31\end{array}$ & $\begin{array}{l}7.02 \\
7.22\end{array}$ \\
\hline $\mathrm{CuCN}\left({ }^{1} \Sigma^{+}\right)$ & $\begin{array}{l}2257 \\
2216\end{array}$ & $\begin{array}{l}0.20 \\
2.52\end{array}$ & $\begin{array}{l}463 \\
461\end{array}$ & $\begin{array}{l}12.61 \\
21.52\end{array}$ & $\begin{array}{l}251 \\
235\end{array}$ & $\begin{array}{l}2.81 \\
5.53\end{array}$ & $\begin{array}{l}7.07 \\
7.47\end{array}$ \\
\hline $\mathrm{ZnCN}\left({ }^{2} \Sigma^{+}\right)$ & 2263 & 24.85 & 397 & 50.25 & 181 & 3.04 & 4.51 \\
\hline & 2126 & 56.01 & 413 & 55.87 & 197 & 1.70 & 4.47 \\
\hline
\end{tabular}

${ }^{\text {a }}$ Note that non-degenerate $\pi$ vibrational frequencies, corresponding to the two Renner-Teller components, are obtained in this case. 
Table S2. Harmonic vibrational frequencies, IR intensities, and dipole moments for MNC species obtained with the B3LYP/6-311+G(3df) and QCISD/6-311+G(d) (second line) methods.

\begin{tabular}{|c|c|c|c|c|c|c|c|}
\hline & \multicolumn{6}{|c|}{ IR frequencies $\left(\mathrm{cm}^{-1}\right)$ and intensities $\left(\mathrm{km} \mathrm{mol}^{-1}\right)$} & \multirow{2}{*}{$\begin{array}{c}\text { Dipole moment } \\
\mu \text { (D) }\end{array}$} \\
\hline & $\begin{array}{c}v_{1} \\
(\mathrm{C}-\mathrm{N} \text { stretch })\end{array}$ & $\mathrm{I}_{1}$ & $\begin{array}{c}v_{2} \\
(\mathrm{M}-\mathrm{N} \text { stretch) }\end{array}$ & $\mathrm{I}_{2}$ & $\begin{array}{c}v_{3} \\
\text { (bend) }\end{array}$ & $\mathrm{I}_{3}$ & \\
\hline $\operatorname{ScNC}\left({ }^{3} \Delta\right)$ & $\begin{array}{l}2114 \\
2086\end{array}$ & $\begin{array}{l}402.93 \\
279.07\end{array}$ & $\begin{array}{l}467 \\
437\end{array}$ & $\begin{array}{l}95.52 \\
85.44\end{array}$ & $\begin{array}{c}115 \\
95\end{array}$ & $\begin{array}{l}0.02 \\
0.00\end{array}$ & $\begin{array}{c}6.02 \\
5.72\end{array}$ \\
\hline $\operatorname{TiNC}\left({ }^{4} \Phi\right)^{\mathrm{a}}$ & $\begin{array}{l}2105 \\
2086\end{array}$ & $\begin{array}{l}341.32 \\
258.19\end{array}$ & $\begin{array}{l}476 \\
450\end{array}$ & $\begin{array}{l}85.32 \\
67.94\end{array}$ & $\begin{array}{c}146,148 \\
122\end{array}$ & $\begin{array}{c}0.34,0.41 \\
0.13\end{array}$ & $\begin{array}{l}6.25 \\
5.88\end{array}$ \\
\hline $\operatorname{VNC}\left({ }^{5} \Delta\right)$ & $\begin{array}{l}2109 \\
2091\end{array}$ & $\begin{array}{l}250.17 \\
224.89\end{array}$ & $\begin{array}{l}475 \\
465\end{array}$ & $\begin{array}{l}65.06 \\
56.20\end{array}$ & $\begin{array}{l}165 \\
139\end{array}$ & $\begin{array}{l}0.90 \\
0.84\end{array}$ & $\begin{array}{l}6.64 \\
6.36\end{array}$ \\
\hline $\operatorname{CrNC}\left({ }^{6} \Sigma^{+}\right)$ & $\begin{array}{l}2121 \\
2099\end{array}$ & $\begin{array}{l}252.82 \\
268.65\end{array}$ & $\begin{array}{l}468 \\
466\end{array}$ & $\begin{array}{c}73.45 \\
101.30\end{array}$ & $\begin{array}{l}161 \\
135\end{array}$ & $\begin{array}{l}2.04 \\
4.86\end{array}$ & $\begin{array}{l}7.38 \\
8.07\end{array}$ \\
\hline $\operatorname{MnNC}\left({ }^{7} \Sigma^{+}\right)$ & $\begin{array}{l}2113 \\
2093\end{array}$ & $\begin{array}{l}380.60 \\
302.97\end{array}$ & $\begin{array}{l}448 \\
437\end{array}$ & $\begin{array}{l}120.63 \\
119.60\end{array}$ & $\begin{array}{l}99 \\
91\end{array}$ & $\begin{array}{l}1.65 \\
0.44\end{array}$ & $\begin{array}{l}4.65 \\
5.01\end{array}$ \\
\hline $\mathrm{FeNC}\left({ }^{6} \Delta\right)$ & $\begin{array}{l}2106 \\
2093\end{array}$ & $\begin{array}{l}435.43 \\
341.85\end{array}$ & $\begin{array}{l}471 \\
466\end{array}$ & $\begin{array}{l}114.29 \\
118.20\end{array}$ & $\begin{array}{l}110 \\
107\end{array}$ & $\begin{array}{l}1.73 \\
0.44\end{array}$ & $\begin{array}{l}4.02 \\
4.47\end{array}$ \\
\hline $\operatorname{CoNC}\left({ }^{3} \Phi\right)^{\mathrm{a}}$ & $\begin{array}{l}2129 \\
2108\end{array}$ & $\begin{array}{l}205.36 \\
230.42\end{array}$ & $\begin{array}{l}482 \\
475\end{array}$ & $\begin{array}{l}37.24 \\
53.14\end{array}$ & $\begin{array}{c}167 \\
108,142\end{array}$ & $\begin{array}{c}4.73 \\
2.84,0.08\end{array}$ & $\begin{array}{l}7.19 \\
7.07\end{array}$ \\
\hline $\operatorname{NiNC}\left({ }^{2} \Sigma^{+}\right)$ & $\begin{array}{l}2148 \\
2118\end{array}$ & $\begin{array}{l}144.52 \\
164.86\end{array}$ & $\begin{array}{l}491 \\
484\end{array}$ & $\begin{array}{l}22.24 \\
43.77\end{array}$ & $\begin{array}{l}167 \\
158\end{array}$ & $\begin{array}{l}0.79 \\
3.87\end{array}$ & $\begin{array}{l}7.38 \\
7.99\end{array}$ \\
\hline $\operatorname{CuNC}\left({ }^{1} \Sigma^{+}\right)$ & $\begin{array}{l}2153 \\
2118\end{array}$ & $\begin{array}{l}156.06 \\
223.83\end{array}$ & $\begin{array}{l}490 \\
497\end{array}$ & $\begin{array}{l}19.73 \\
46.98\end{array}$ & $\begin{array}{l}152 \\
141\end{array}$ & $\begin{array}{l}2.25 \\
4.16\end{array}$ & $\begin{array}{l}7.17 \\
7.55\end{array}$ \\
\hline $\mathrm{ZnNC}\left({ }^{2} \Sigma^{+}\right)$ & 2141 & 256.07 & 444 & 54.99 & 103 & 0.40 & 4.65 \\
\hline & 2107 & 274.84 & 460 & 80.21 & 109 & 0.07 & 4.77 \\
\hline
\end{tabular}

${ }^{a}$ Note that non-degenerate $\pi$ vibrational frequencies, corresponding to the two Renner-Teller components, are obtained in this case. 
Table S3. Optimized geometries ( $\AA$ and degrees) of the transition states for the cyanideisocyanide isomerization obtained with the B3LYP/6-311+G(3df) and QCISD/6-311+G(3df) (second line) methods. QCISD vibrational frequencies have been computed with the 6$311+\mathrm{G}(\mathrm{d})$ basis set.

\begin{tabular}{llllll}
\hline & \multicolumn{3}{c}{ Geometry } & Vibrational frequencies \\
\cline { 2 - 5 }$\left(\mathrm{cm}^{-1}\right)$
\end{tabular}


Table S4. Energy decomposition analysis ( $\mathrm{kcal} / \mathrm{mol})$ of $\mathrm{M}-\mathrm{CN}$ and $\mathrm{M}-\mathrm{NC}(\mathrm{M}=\mathrm{Sc}, \mathrm{Ti}, \mathrm{V}, \mathrm{Cr}, \mathrm{Mn})$ at the BP86/TZ2P+ level of theory.

\begin{tabular}{|c|c|c|c|c|c|c|c|c|c|c|c|}
\hline \multirow{2}{*}{ TM-CN } & & \multicolumn{2}{|c|}{$\operatorname{Sc}\left({ }^{3} \Delta\right)$} & \multicolumn{2}{|c|}{$\operatorname{Ti}\left({ }^{4} \Phi\right)$} & \multicolumn{2}{|c|}{$\mathrm{V}\left({ }^{5} \Delta\right)$} & \multicolumn{2}{|c|}{$\operatorname{Cr}\left({ }^{6} \Sigma^{+}\right)$} & \multicolumn{2}{|c|}{$\operatorname{Mn}\left({ }^{7} \Sigma^{+}\right)$} \\
\hline & $\Delta \mathrm{E}_{\mathrm{int}}$ & -171.9 & & -188.7 & & -199.8 & & -186.6 & & -172.9 & \\
\hline & $\Delta \mathrm{E}_{\text {pauli }}$ & 134.7 & & 138.4 & & 154.6 & & 137.9 & & 125.5 & \\
\hline & $\Delta \mathrm{E}_{\text {elstat }}$ & -212.9 & $(69.5 \%)^{\mathrm{a}}$ & -233.9 & $(71.5 \%)$ & -250.3 & $(70.6 \%)$ & -245.6 & $(75.7 \%)$ & -228.3 & $(76.5 \%)$ \\
\hline & $\Delta \mathrm{E}_{\text {orb }}$ & -93.7 & $(30.5 \%)$ & -93.2 & $(28.5 \%)$ & -104.1 & $(29.4 \%)$ & -78.9 & $(24.3 \%)$ & -70.2 & $(23.5 \%)$ \\
\hline & $\Delta \mathrm{E}(\sigma)$ & -78.6 & $(83.9 \%)^{b}$ & -69.3 & $(74.4 \%)$ & -84.3 & $(81.0 \%)$ & -60.0 & $(76.0 \%)$ & -53.2 & $(75.8 \%)$ \\
\hline & $\Delta \mathrm{E}(\pi)$ & -13.9 & $(14.9 \%)$ & -23.2 & $(24.9 \%)$ & -19.4 & $(18.6 \%)$ & -18.8 & $(23.9 \%)$ & -17.0 & $(24.2 \%)$ \\
\hline & $\Delta \mathrm{E}(\delta)$ & -1.2 & $(1.2 \%)$ & -0.7 & $(0.7 \%)$ & -0.4 & $(0.4 \%)$ & -0.1 & $(0.1 \%)$ & 0.0 & $(0.0 \%)$ \\
\hline \multirow[t]{7}{*}{ TM-NC } & $\Delta \mathrm{E}_{\text {int }}$ & -175.5 & & -189.4 & & -196.3 & & -182.0 & & -171.7 & \\
\hline & $\Delta \mathrm{E}_{\text {pauli }}$ & 158.8 & & 149.1 & & 150.7 & & 144.8 & & 132.3 & \\
\hline & $\Delta \mathrm{E}_{\text {elstat }}$ & -226.4 & $(67.7 \%)$ & -235.2 & $(69.5 \%)$ & -241.9 & $(69.7 \%)$ & -238.8 & $(73.1 \%)$ & -228.5 & $(75.2 \%)$ \\
\hline & $\Delta \mathrm{E}_{\text {orb }}$ & -107.8 & $(32.3 \%)$ & -103.3 & $(30.5 \%)$ & -105.2 & $(30.3 \%)$ & -87.9 & $(26.9 \%)$ & -75.5 & $(24.8 \%)$ \\
\hline & $\Delta \mathrm{E}(\sigma)$ & -82.6 & $(76.5 \%)$ & -65.2 & $(63.1 \%)$ & -77.2 & $(73.5 \%)$ & -62.2 & $(70.8 \%)$ & -49.4 & $(65.4 \%)$ \\
\hline & $\Delta \mathrm{E}(\pi)$ & -24.2 & $(22.4 \%)$ & -37.4 & $(36.2 \%)$ & -27.5 & $(26.2 \%)$ & -25.7 & $(29.2 \%)$ & -26.1 & $(34.6 \%)$ \\
\hline & $\Delta \mathrm{E}(\delta)$ & -1.1 & $(1.1 \%)$ & -0.7 & $(0.7 \%)$ & -0.4 & $(0.4 \%)$ & 0.0 & $(0.0 \%)$ & 0.0 & $(0.0 \%)$ \\
\hline
\end{tabular}

${ }^{\text {a }}$ Values in parentheses give the percentage of attractive interactions $\Delta \mathrm{E}_{\text {elstat }}+\Delta \mathrm{E}_{\text {orb }}$.

${ }^{\mathrm{b}}$ Values in parentheses give the percentage contribution to the total orbital interactions. 
Table S5. Energy decomposition analysis (kcal/mol) of M-CN and M-NC (M=Fe, Co, Ni, Cu, Zn) at the BP86/TZ2P+ level of theory.

\begin{tabular}{|c|c|c|c|c|c|c|c|c|c|c|c|}
\hline \multirow{2}{*}{ TM-CN } & \multirow[b]{2}{*}{$\Delta \mathrm{E}_{\mathrm{int}}$} & \multicolumn{2}{|c|}{$\operatorname{Fe}\left({ }^{6} \Delta\right)$} & \multicolumn{2}{|c|}{$\operatorname{Co}\left({ }^{3} \Delta\right)$} & \multicolumn{2}{|c|}{$\left.\mathrm{Ni}^{2} \Delta\right)^{\mathrm{c}}$} & \multicolumn{2}{|c|}{$\mathrm{Cu}\left({ }^{1} \Sigma^{+}\right)$} & \multicolumn{2}{|c|}{$\mathrm{Zn}\left({ }^{2} \Sigma^{+}\right)$} \\
\hline & & -185.8 & & -207.8 & & -214.1 & & -212.9 & & -191.6 & \\
\hline & $\Delta \mathrm{E}_{\text {pauli }}$ & 138.7 & & 157.1 & & 163.6 & & 157.5 & & 133.7 & \\
\hline & $\Delta \mathrm{E}_{\text {elstat }}$ & -241.7 & $(74.5 \%)^{\mathrm{a}}$ & -284.1 & $(77.8 \%)$ & -295.2 & $(78.2 \%)$ & -292.8 & $(79.0 \%)$ & -242.6 & $(74.6 \%)$ \\
\hline & $\Delta \mathrm{E}_{\text {orb }}$ & -82.8 & $(25.5 \%)$ & -80.9 & $(22.2 \%)$ & -82.5 & $(21.8 \%)$ & -77.6 & $(21.0 \%)$ & -82.7 & $(25.4 \%)$ \\
\hline & $\Delta \mathrm{E}(\sigma)$ & -60.9 & $(73.7 \%)^{b}$ & -52.1 & $(64.4 \%)$ & -58.3 & $(70.7 \%)$ & -54.2 & $(69.8 \%)$ & -67.5 & $(81.6 \%)$ \\
\hline & $\Delta \mathrm{E}(\pi)$ & -20.8 & $(25.1 \%)$ & -28.0 & $(34.7 \%)$ & -24.2 & $(29.3 \%)$ & -23.2 & $(29.8 \%)$ & -14.9 & $(18.1 \%)$ \\
\hline & $\Delta \mathrm{E}(\delta)$ & -1.0 & $(1.2 \%)$ & -0.8 & $(0.9 \%)$ & 0.0 & $(0.0 \%)$ & -0.3 & $(0.4 \%)$ & -0.3 & $(0.3 \%)$ \\
\hline \multirow[t]{7}{*}{ TM-NC } & $\Delta \mathrm{E}_{\text {int }}$ & -185.2 & & -194.3 & & -199.3 & & -198.5 & & -184.3 & \\
\hline & $\Delta \mathrm{E}_{\text {pauli }}$ & 146.1 & & 134.4 & & 131.4 & & 139.0 & & 126.1 & \\
\hline & $\Delta \mathrm{E}_{\text {elstat }}$ & -242.0 & $(73.0 \%)$ & -251.3 & $(76.5 \%)$ & -254.0 & $(76.8 \%)$ & -261.1 & $(77.4 \%)$ & -230.6 & $(74.3 \%)$ \\
\hline & $\Delta \mathrm{E}_{\text {orb }}$ & -89.3 & $(27.0 \%)$ & -77.3 & $(23.5 \%)$ & -76.6 & $(23.2 \%)$ & -76.3 & $(22.6 \%)$ & -79.8 & $(25.7 \%)$ \\
\hline & $\Delta \mathrm{E}(\sigma)$ & -56.8 & $(63.6 \%)$ & -47.2 & $(61.0 \%)$ & -46.9 & $(61.2 \%)$ & -50.5 & $(66.2 \%)$ & -59.6 & $(74.8 \%)$ \\
\hline & $\Delta \mathrm{E}(\pi)$ & -31.5 & $(35.2 \%)$ & -29.4 & $(38.0 \%)$ & -28.4 & $(37.1 \%)$ & -25.5 & $(33.5 \%)$ & -19.8 & $(24.9 \%)$ \\
\hline & $\Delta \mathrm{E}(\delta)$ & -1.0 & $(1.2 \%)$ & -0.8 & $(1.0 \%)$ & -1.4 & $(1.7 \%)$ & -0.3 & $(0.3 \%)$ & -0.3 & $(0.3 \%)$ \\
\hline
\end{tabular}

${ }^{\mathrm{a}}$ Values in parentheses give the percentage of attractive interactions $\Delta \mathrm{E}_{\text {elstat }}+\Delta \mathrm{E}_{\text {orb }}$

${ }^{\mathrm{b}}$ Values in parentheses give the percentage contribution to the total orbital interactions.

c Values for the NiNC isomer correspond to the ${ }^{2} \Sigma^{+}$state. 


\section{Complete Reference 47:}

Frisch, M. J., Trucks, G. W., Schlegel, H. B.; Scuseria, G. E.; Robb, M. A.; Cheeseman, J. R.; Zakrzewski, V. G.; Montgomery Jr. J. A.; Stratmann, R. E.; Burant, J. C.; Dapprich, S.; Millan, J. M.; Daniels, A. D.; Kudin, K. N.; Strain, M. C.;. Farkas, O.; Tomasi, J.; Barone, V.; Cossi, M.; Cammi, R.; Mennucci, B.; Pomelly, C.; Adamo, C.; Clifford, S.; Ochterski, J.; Petersson, G. A.; Ayala, P. Y.; Cui, Q.; Morokuma, K.; Malick, D. K.; Rabuck, A. D.; Raghavachari, K.; Foresman, J. B.; Cioslowski, J.; Ortiz, J. V.; Baboul, A. G.; Stefanov, B. B.; Liu, G.; Liashenko, A.; Piskorz, P.; Komaromi, I.; Gomperts, R.; Martin, R. L.; Fox, D. J.; Keith, T.; Al-Laham, M. A.; Peng, C. Y.; Nanayakkara, A.; Gonzalez, C.; Challacombe, M.; Gill, P. M. W.; Johnson, B.; Chen, W.; Wong, M. W.; Andres, J. L.; Gonzalez, C.; Head-Gordon, M.; Replogle, E. S.; Pople, J. A. Gaussian 98, Gaussian Inc, Pittsburgh, PA, 1998.

\section{Complete Reference 52:}

MOLPRO, a package of ab initio programs designed by H.-J. Werner and P. J. Knowles, version 2002.1, Amos, R. D.; Bernhardsson, A.; Berning, A.; Celani, P.; Cooper, D. L.; Deegan, M. J. O.; Dobbyn, A. J.; Eckert, F.; Hampel, C.; Hetzer, G.; Knowles, P. J.; Korona, T.; Lindh, R.; Lloyd, A.W.; McNicholas, S. J.; Manby, F. R.; Meyer, W.; Mura, M. E.; Nicklass, A.; Palmieri, P.; Pitzer, R.; Rauhut, G.; Schütz, M.; Schumann, U.; Stoll, H.; Stone, A. J.; Tarroni, R.; Thorsteinsson, T.; Werner., H.-J., 2002. 